\section{Metabolism of Alpha-carotene}

IN the course of an earlier investigation into the utilization of carotene by the rat as a source of vitamin A, it was noted that there was a consistent difference between the absorption spectra of the liver non-saponifiable fractions from those rats fed vitamin $\mathrm{A}$ and those fed a mixture of 90 per cent beta- and 10 per cent alpha-carotene ${ }^{1}$. This difference was in the magnitude of the absorbance at $311 \mathrm{~m} \mu$ as compared with that at $326 \mathrm{~m} \mu$. The alphaionone analogue of vitamin A was afterwards synthesized ${ }^{2}$ and the absorption maxima were found to be at $311 \mathrm{~m} \mu$ $\left(E_{1 \mathrm{~cm}}^{1 \%}, 1,650\right)$ and $325 \mathrm{~m} \mu\left(E_{1 \mathrm{~cm}}^{1 \%}, 1,500\right)$. This alpha-vitamin A was found to be biologically inactive but was found to be stored in the liver in the same way as vitamin A (ref. 3).

Tho purpose of the investigation reported here was to ascertain whether the abnormalities in the absorption spectra noted with rats fed a mixture of alpha- and betacarotene could be the result of the deposition of alphavitamin $\mathbf{A}$ in the liver.

Female Sprague-Dawley rats with nursing litters were placed on a purified diet when the young were about 10 days old. Their dietary materials were so chosen that the basic ration was nutritionally complete except for the essential fatty acids and the fat-soluble vitamins. The fat-soluble vitamins were given dropwise by mouth, one drop twice each week. The weekly supplement contained 50 U.S.P. units of vitamin A, 100 U.S.P. units of vitamin $\mathrm{D}$ and $3.0 \mathrm{mg}$ of alpha-tocopherol. This level of vitamin A intake allowed normal growth but did not result in any measurable liver storage of the vitamin. Half the animals were allowed to become depleted in the essential fatty acids, while cottonseed oil was added to the basal diet of the remainder of the animals in suffieient quantity to satisfy the requirement for the essential fatty acids. Each group was divided into three parts and the diets were supplemented with either vitamin A, beta-carotene or alpha-carotene when deficiency symptoms appeared in the animals depleted in essential fatty acids. After a 10-day supplementation period, during which the animals received $100 \mathrm{~g}$ of alpha- or beta-carotene, or 250 U.S.P. units of vitamin A per day, the animals were killed. The livers were removed, and the non-saponifiable extracts were dissolved in cyclohexane. The absorption spectrum was determined for each solution, and a sample was then exposed to ultra-violet light for a period suffioiently Iong to effect the complete destruction of any photosensitive material. This was presumed to include both vitamin A and alpha-vitamin A. A second absorption spectrum was obtained over the same spectral range. The differenee between the two curves obtained was thought to give a truer picture of the vitamin A content of the extracts, since the non-specific absorption is not uniform over the spectral range investigated.

The alpha- and beta-carotene were examined spectrophotometrically to make certain that they were not mixtures of the alpha and beta forms.

A comparison of typical absorption spectra of the photosensitive materials stored in the livers, and extracted with the non-saponifiable fraction, is shown in Fig. 1. These three animals were females fed on diet I. As can be soen in the figure, the absorption spectra show no significant difference when the animals are fed vitamin A or beta-carotene. There was no reason to expect that there would be any difference. However, when the same comparison is made with the extracts from animals fed vitamin A or alpha-carotene, the results are manifestly

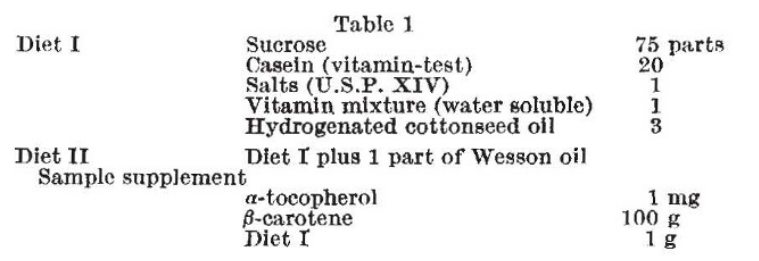

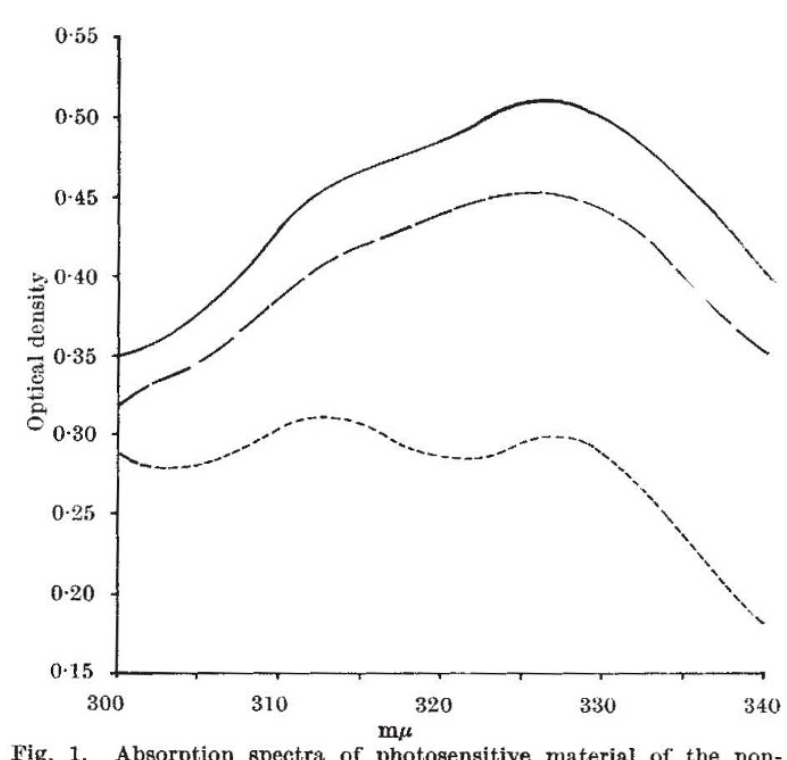

Fig. 1. Absorption spectra of photosensitive material of the non-

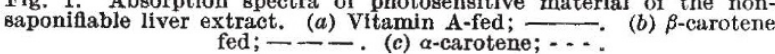

different. It is true that the material deposited in the liver with alpha-carotene feeding is not all alpha-vitamin A. The ratio $E_{311} / E_{326}$ found for the pure compound was $1 \cdot 10$ (ref. 2), while for the curve shown in Fig. 1 the ratio is 1.02. It is obvious that the liver extract from the animal fed alpha-carotene contained more alpha-vitamin A than true vitamin $\mathbf{A}$ since the corresponding ratio for the vitamin $A$ fed animal is $0 \cdot 86$, and this same value was found for the natural vitamin A concentrate used as a supplement.

When the quantities of vitamin $\mathrm{A}$ and alpha-vitamin $\mathrm{A}$ are calculated from the data shown in the figure using the $E_{1 \% \mathrm{~m}}^{1 \%}$ values at $311 \mathrm{~m} \mu$ and $326 \mathrm{~m} \mu$ for the pure compounds, it is found that the liver contained $29 \mathrm{~g}$ of vitamin $\mathrm{A}$ and $67 \mathrm{~g}$ of alpha-vitamin A. Since the animal gained weight during the supplementation period, it seems safe to assume that some vitamin A was utilized for growth. If this allowance is made, the quantity of alphavitamin $\mathrm{A}$ is approximately equal to the quantity of vitamin A which must have boen produced.

The feeding of alpha-carotene resulted, then, in the formation of a different end-product than did the feeding of either vitamin A or beta-carotene.

The results of this investigation do not unequivocally support either the theory of oxidation or hydrolysis at the central double bond of the carotene molecule ${ }^{4,5}$ or the proposed mechanism of an oxidative process beginning at one end of the molecule ${ }^{6}$. What is demonstrated is that whatever the mechanism it very probably results in the formation of two products-vitamin A and alpha-vitamin A - and the storage of both in the liver of the test animal.

This work was completed at the University of Miami, Coral Gables, Florida, and was sponsored by the Research Corporation.

\section{Occidental College, Los Angeles, California.}

\section{J. S. McAnally}

\section{D. SZYManski}

National Starch and Chemical Corporation,

Plainfield, New Jersey.

${ }^{1}$ MeAnally, J. S., thesis, Univ. Indiana (1950). 2 Robeson, C. D., Cawley, J. D., Weisler, L., Stern, M. H., Eddinger, C. C.,
and Chechak, A. J., J. Amer. Chem. Soc., Yry, 4111 (1955).

${ }^{3}$ Ames, S. R., Swanson, W. J., and Harris, P. L., J. Amer. Chem. Soc., 77, 4136 (1955).

${ }^{4}$ Hunter, R. F., Nature, 158, 257 (1946)

' Karrer, P., Morf, R., and Schöpp, K., Helv. Chim. Acta, 14, 1036, 1431 (1931).

'Redfearn, E. R., Biochem. J., 66, 39P (1957).

'McAnally, J. S., and Szymanski, C. D., Fed. Proc., 16, 219 (1957). 